\title{
Brain Signal Based Driver Drowsiness Prediction
}

\section{Addzrull Hi-Fi Syam Ahmad Jamil, Mohd Lutfi Mohd Khidir, Mohd Firdaus Mohd Mokhtar}

To Link this Article: http://dx.doi.org/10.6007/IJARBSS/v11-i2/8616

DOI:10.6007/IJARBSS/v11-i2/8616

Received: 17 December 2020, Revised: 12 January 2021, Accepted: 25 January 2021

Published Online: 09 February 2021

In-Text Citation: (Jamil et al., 2021)

To Cite this Article: Jamil, A. H. S. A., Khidir, M. L. M., \& Mokhtar, M. F. M. (2021). Brain Signal Based Driver Drowsiness Prediction. International Journal of Academic Research in Business and Social Sciences, 11(2), 75-80.

\section{Copyright: (c) 2021 The Author(s)}

Published by Human Resource Management Academic Research Society (www.hrmars.com) This article is published under the Creative Commons Attribution (CC BY 4.0) license. Anyone may reproduce, distribute, translate and create derivative works of this article (for both commercial and non-commercial purposes), subject to full attribution to the original publication and authors. The full terms of this license may be seen at: http://creativecommons.org/licences/by/4.0/legalcode

Vol. 11, No. 2, 2021, Pg. 75 - 80

Full Terms \& Conditions of access and use can be found at http://hrmars.com/index.php/pages/detail/publication-ethics 


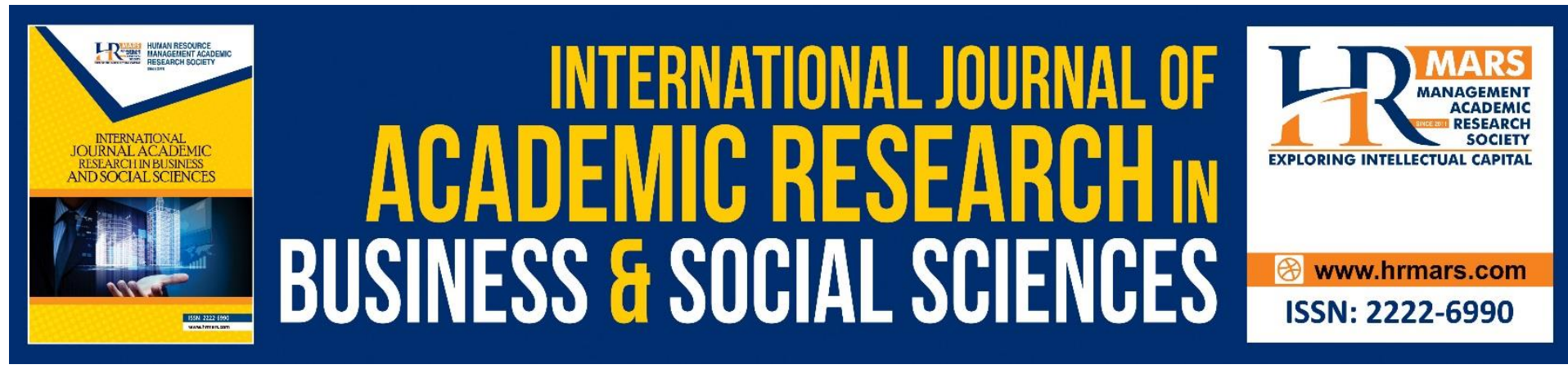

\title{
Brain Signal Based Driver Drowsiness Prediction
}

\author{
Addzrull Hi-Fi Syam Ahmad Jamil \\ Politeknik Kota Kinabalu, Malaysia
}

\author{
Mohd Lutfi Mohd Khidir, Mohd Firdaus Mohd Mokhtar \\ Politeknik Sultan Abdul Halim, Malaysia
}

\begin{abstract}
Drowsiness has been one of the leading causes of injuries or fatalities in vehicle accidents. Therefore, in this research work, it is proposed to develop an adaptive heavy vehicle driver fatigue and alertness model based on EEG frequency bands by combining signal processing algorithms and soft computing techniques such as Neuro-fuzzy algorithm to estimate the driver cognitive state while driving a vehicle in a virtual reality (VR)-based dynamic simulator under monotonous driving environment. Thus in this research, it is proposed to minimize the number of features using soft computing techniques and classification using non-linear supervised classification algorithms. The proposed adaptive model identifies the discrimination between the driver's level of fatigue by recognizing whether the driver is fatigue due to task-induced factors or attitude/behaviour using the brain responses, then the level of fatigue is related with sleepiness (i.e. level of alertness towards driving). Further, the adaptive model can be utilized to alert drivers and regulators in optimizing the properties of the interface systems in identifying potential catastrophe. The proposed system alerts the driver during fatigue/drowsiness according to the recognition of cognitive state and produce the fatigue index and level of alertness. The proposed system also helps the driver to be more attentive and intuitive to prevent from fatal road accidents.
\end{abstract}

Keywords: $\mathrm{BCl}, \mathrm{EEG}$, Drowsiness, VR, Neuro-fuzzy Algorithm

\section{Introduction}

Fatigue, sleepiness and stress while driving are common among the heavy vehicle and car drivers in Malaysia, with various possible causes: acute and chronic sleep deprivation, driving the vehicle for long hours and at different driving patterns, irregular schedule changes, and sleep disorders due to the driver's working conditions, especially at monotonous driving environment (Al-Sultan, Al-Bayatti, \& Zedan, 2013; Fai, 2015).

Driving a vehicle under the influences of fatigue/drowsiness will cause longer response time, vigilance reduction and deficits in communication and information processing, which may lead to higher risk of collision and lacks correctness in decision-making, especially at high speeds. Statistics on fatal or injury-causing traffic accidents by Malaysian Institute of Road Safety Research (MIROS) shows that the death-to-population ratio stands at 23.8 to 100,000 Malaysian people, $80 \%$ of fatal accidents are due to human errors (Mustafa, 2015). 
The available technologies for monitoring the driver's cognitive state are still in its infancy and the knowledge of understanding government policies (JPJ, PDRM, JKJR, MIROS, $\mathrm{JKR}$ ) focusing on $4 \mathrm{E}$ (Engineering, Enforcement, Education and Environment) and vehicle manufacturer's strategies are yet not sufficient to prevent from fatal road accidents (Jonathan, 2015).

In recent years, a variety of methods and approaches have been proposed by researchers for detecting driver fatigue/drowsiness based on eye movements, head movements and bio signals. Among the various psychophysiological based approaches as indicative measure, EEG perhaps being the most promising indicators of driver fatigue. However, there are some challenges in developing EEG based driver alertness systems, which include, lack of significant index for detecting fatigue and pervasive noise interferences while acquiring the EEG signals in a realistic and dynamic driving environment (Liang et al., 2005).

Furthermore, driver fatigue may also due to task-induced factors such as high density of traffic, body posture and under exposure to vibration and noise that may not relate with sleepiness. At present, there is no adaptive model to discriminate the correlation between the physical and cognitive consequences of fatigue that relates with driver alertness. Hence, it is necessary to develop an adaptive driver fatigue identification model considering various environmental and behavioral aspects of the driver and evaluate the level of alertness (Touryan et al., 2016).

The Event-related potentials (ERP) observed from the drivers can be used to identify the cognitive state and fatigue index that may improve the driver's performance capacity and prevents from catastrophic incident. However, there are limited information on the correlation between driver fatigues due to task-induced factors and attitude/behaviour. Therefore, understanding the psychology of fatigue may lead to better fatigue-alertness model (Yang, Lin, \& Bhattacharya, 2010).

\section{Literature Review}

Heavy vehicles (E-class license) driver fatigue is a major exogenous cause of road accidents and has implications for Malaysian road safety (Lal, Craig, Boord, Kirkup, \& Nguyen, 2003; Mustafa, 2015). Statistics on fatal road accidents by Malaysian Institute of Road Safety Research (MIROS) shows that the death-to-population ratio stands at 23.8 to 100,000 people (10.3\% (lorry) and 1.59 \% (Bus)), compared to the world average of 18 to 100,000 people, human errors being $80 \%$ (Jonathan, 2015). The major aspect that causes human errors are fatigue or drowsiness due to personality and temperament, lack of sleep, consumption of alcohol, long driving hours and driving patterns such as driving at midnight, early dawn, midafternoon hours and especially in the monotonous driving environment, personality and temperament may also influence fatigue (Stern, Boyer, \& Schroeder, 1994; Wang \& Wets, 2013). Therefore, preventing such catastrophic accidents is thus a major focus of government policies, vehicle manufactures strategies and research efforts in the field of automotive and safety research(Charbonnier, Roy, Bonnet, \& Campagne, 2016; Mustafa, 2015; Wang \& Wets, 2013).

In recent years, there are number of techniques and approaches that have been proposed to recognize vigilance changes in the past such as physical changes during fatigue and measuring physiological changes of drivers, such as eye activity measurement, heart beat rate, skin electric potential, and especially, brain wave activities as a means of detecting the cognitive states (Al-Sultan et al., 2013; Lee \& Chung, 2012; Liang et al., 2005). Therefore, monitoring EEG signals during driver fatigue may be a promising variable for use in 
fatigue/drowsiness countermeasure systems. EEG based identification of alertness levels have the advantages in making accurate and quantitative assessment and relatively shorter one to track second-to-second fluctuations in the driver's performance.

However, EEG based monitoring fatigue and evaluating the significant index level are still in its infancy and there are lot to explore such as the EEG frequency spindles correlates of fatigue and drowsiness, as well as to evaluate what extent these cognitive-state related EEG activities can be efficiently incorporated into a real-time fatigue monitoring system (Charbonnier et al., 2016; Johnson et al., 2011; Lal et al., 2003; Touryan et al., 2016). Also, there are challenges in processing the EEG signals, which contains pervasive noise interferences while recording the brain responses in a realistic and dynamic driving environment (Yang et al., 2010).

Therefore, in this research work, it is proposed to develop an adaptive heavy vehicle driver fatigue and alertness model based on EEG frequency bands by combining signal processing algorithms and soft computing techniques such as Neuro-fuzzy algorithm to estimate the driver cognitive state while driving a vehicle in a virtual reality (VR)-based dynamic simulator under monotonous driving environment. To minimize the computational time, the features used for modelling should be minimal. Thus in this research, it is proposed to minimize the number of features using soft computing techniques and classification using non-linear supervised classification algorithms. The proposed adaptive model identifies the discrimination between the driver's level of fatigue by recognizing whether the driver is fatigue due to task-induced factors or attitude/behaviour using the brain responses, then the level of fatigue is related with sleepiness (i.e. level of alertness towards driving).

\section{Methodology}

The following are the methods to produce adaptive heavy vehicle fatigue and alertness. EEG signals and Neuro-fuzzy are used to estimate the driver's condition while using the driving simulator. Figure 1.0 is a flow chart regarding the formulation of the methodology performed

\section{Development of a Virtual Reality Driving Simulator}

The development of VR based driving simulation requires broad areas of knowledge and learning. Further, the placement of electrodes for the EEG acqusition corresponding to the cognitive state involves different experimental observations.

\section{Selection of EEG Electrodes, Subjects and Data Acqusition Methods}

Based on the literature, the placement of electrodes for the EEG acquisition corresponding to the cognitive state involves different experimental observations. Based on the number of channels chosen for the experiment, the EEG data acqusition methods have to be chosen. Subjects chosen for this study have to be from similar age groups.

\section{Protocol Development for VR based Driving Experiment}

An EEG data acquisition protocol have to be developed to capture the brain activity while the subject is drives the VR based driving simulator. The subjects have to be given unlimited access to practice the driving simulator and to be checked for health issues, smoking and alcohol consumption before the experiment. The protocol scenario has to involve real life simulations like, lane deviation, parking, driving up hill and driving in dark. Data validation protocols have to developed for validating the recorded EEG signals. 


\section{Algorithm Development for Feature Extraction}

The recorded EEG signals have to be pre-processed to remove artefacts and extract features corresponding to the nature of the activity. The feature extraction algorithms have to be applied upon the EEG samples to produce different data sets.

\section{Model Developement for Prediction of the Driver Drowsiness}

A generalized model have to be developed based on deep learning neural network to predict the drowsiness of the driver. The dataset prepared fom the feature extraction process has to be verified and validated for misssing or ourliers. Prominent features will be extracted from the dataset using feature selection and optimization algorithms. Then the datasets generated from the EEG signals during the VR based driving experiment have to be mapped with the corresponding drowsiness index

\section{Testing, Validation and Hardware Development}

The developed models have to be validated against the validation dataset for accuracy. A microcontroller based hardware setup has to be developed to demonstrate the working model of the developed prediction model.

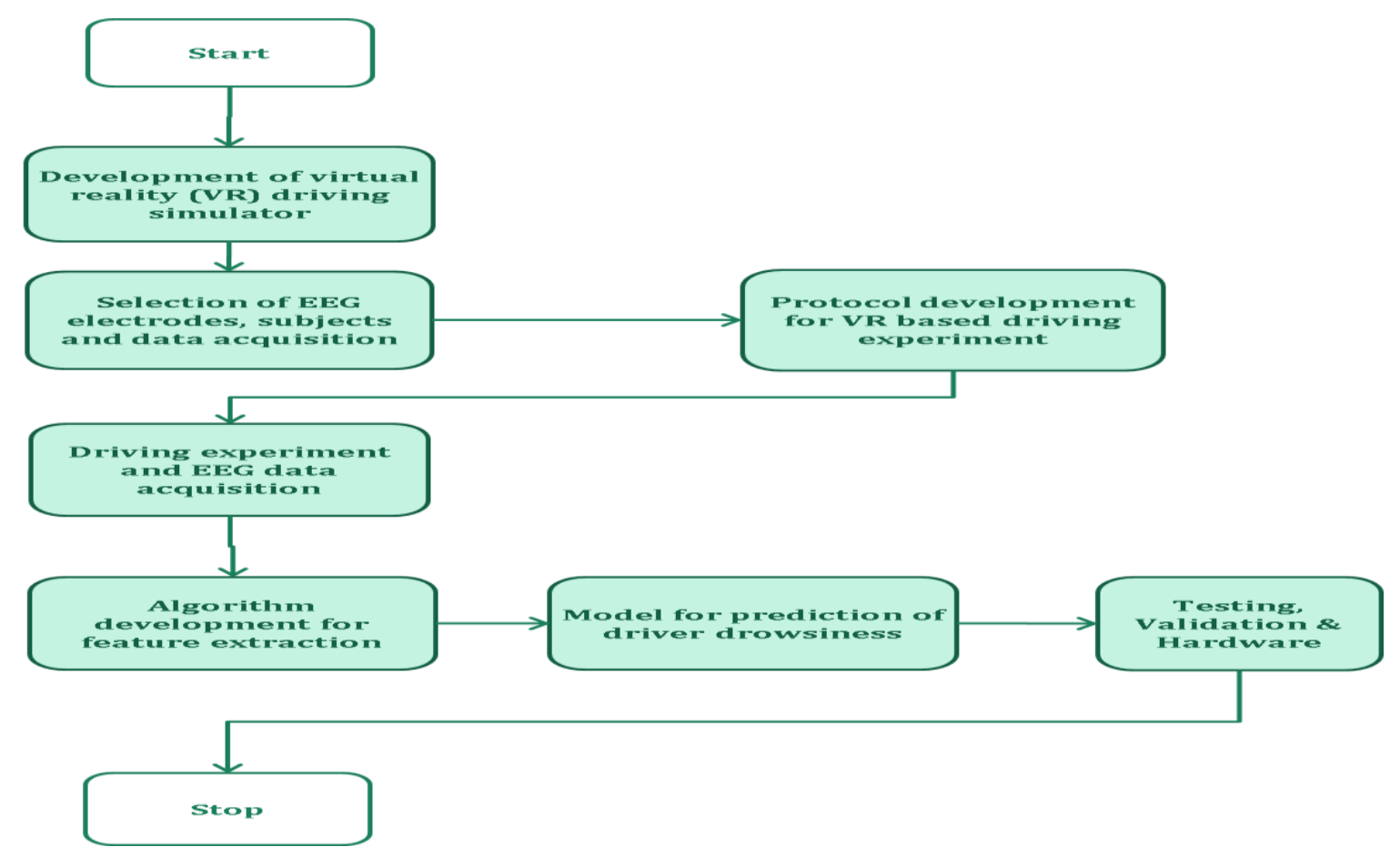

\subsection{A flowchart to show the methodology operation steps of Brain Signal Based Driver Drowsiness Prediction.}

\section{Conclusion}

The adaptive model can be utilized to alert drivers and regulators in optimizing the properties of the interface systems in identifying potential catastrophe. The proposed system alerts the driver during fatigue/drowsiness according to the recognition of cognitive state and produce the fatigue index and level of alertness. The proposed system also helps the driver to be more attentive and intuitive to prevent from fatal road accidents. 


\section{Corresponding Author}

Addzrull Hi-Fi Syam, Politeknik Kota Kinabalu, Malaysia

Email: azrulhifisyam@yahoo.com

\section{References}

Al-Sultan, S., Al-Bayatti, A. H., \& Zedan, H. (2013). Context-Aware Driver Behavior Detection System in Intelligent Transportation Systems. IEEE Transactions on Vehicular Technology, 62(9), 4264-4275. https://doi.org/10.1109/TVT.2013.2263400

Charbonnier, S., Roy, R. N., Bonnet, S., \& Campagne, A. (2016). EEG index for control operators' mental fatigue monitoring using interactions between brain regions. Expert Systems with Applications, 52, 91-98. https://doi.org/10.1016/j.eswa.2016.01.013

Fai, L. C. (2015). Miros statistics say human error causes $80 \%$ of traffic accidents. The Sun Daily. Retrieved from https://www.thesundaily.my/archive/1333889-KRARCH296470

Johnson, R. R., Popovic, D. P., Olmstead, R. E., Stikic, M., Levendowski, D. J., \& Berka, C. (2011). Drowsiness/alertness algorithm development and validation using synchronized EEG and cognitive performance to individualize a generalized model. Biological Psychology, 87(2), 241-250. https://doi.org/10.1016/j.biopsycho.2011.03.003

Jonathan, L. (2015). Road deaths in Malaysia above world average - report. Retrieved from https://paultan.org/2015/10/13/road-deaths-in-malaysia-above-world-average-report/

Lal, S. K. L., Craig, A., Boord, P., Kirkup, L., \& Nguyen, H. (2003). Development of an algorithm for an EEG-based driver fatigue countermeasure. Journal of Safety Research, 34(3), 321328. https://doi.org/10.1016/S0022-4375(03)00027-6

Lee, B.-G., \& Chung, W.-Y. (2012). Driver Alertness Monitoring Using Fusion of Facial Features and Bio-Signals. IEEE Sensors Journal, 12(7), 2416-2422. https://doi.org/10.1109/JSEN.2012.2190505

Liang, S. F., Lin, C. T., Wu, R. C., Chen, Y. C., Huang, T. Y., \& Jung, T. P. (2005). Monitoring Driver's Alertness Based on the Driving Performance Estimation and the EEG Power Spectrum Analysis. 2005 IEEE Engineering in Medicine and Biology 27th Annual Conference, 5738-5741. https://doi.org/10.1109/IEMBS.2005.1615791

Mustafa, M. N. (2015). Overview Of Current Road Safety Situation In Malaysia. First International Visual Informatics Conference.

Stern, J. A., Boyer, D., \& Schroeder, D. (1994). Blink Rate: A Possible Measure of Fatigue. Human Factors: The Journal of the Human Factors and Ergonomics Society, 36(2), 285297. https://doi.org/10.1177/001872089403600209

Touryan, J., Lance, B. J., Kerick, S. E., Ries, A. J., \& McDowell, K. (2016). Common EEG features for behavioral estimation in disparate, real-world tasks. Biological Psychology, 114, 93107. https://doi.org/10.1016/j.biopsycho.2015.12.009

Wang, W., \& Wets, G. (Eds.). (2013). Computational Intelligence for Traffic and Mobility. https://doi.org/10.2991/978-94-91216-80-0

Yang, G., Lin, Y., \& Bhattacharya, P. (2010). A driver fatigue recognition model based on information fusion and dynamic Bayesian network. Information Sciences, 180(10), 19421954. https://doi.org/10.1016/j.ins.2010.01.011 